\title{
Carbazole Containing Copolymers: Synthesis, Characterization, and Applications in Reversible Holographic Recording
}

\author{
Bénédicte Mailhot-Jensen, ${ }^{1}$ Stefan Robu, ${ }^{2}$ Agnès Rivaton, ${ }^{1}$ Jean-François Pilichowski, ${ }^{1}$ \\ Arcadi Chirita, ${ }^{2}$ Ekaterina Chilat, ${ }^{1,2}$ and Galina Dragalina ${ }^{2}$ \\ ${ }^{1}$ Clermont Université, Université Blaise Pascal, CNRS, UMR 6505, Laboratoire de Photochimie Moléculaire et Macromoléculaire, \\ 24 avenue des Landais, BP 80026, 63171 Aubière Cedex, France \\ ${ }^{2}$ Moldova State University, 60, A. Mateevici Street, 2009 Chisinau, Moldova
}

Correspondence should be addressed to Bénédicte Mailhot-Jensen, benedicte.mailhot@u-clermont1.fr

Received 9 June 2010; Accepted 22 July 2010

Academic Editor: Mark van Der Auweraer

Copyright (C) 2010 Bénédicte Mailhot-Jensen et al. This is an open access article distributed under the Creative Commons Attribution License, which permits unrestricted use, distribution, and reproduction in any medium, provided the original work is properly cited.

\begin{abstract}
Carbazolic copolymers have been developed to be used in reversible holographic recording. This paper describes a complete analysis, from synthesis of the material to its applications, together with the corresponding characterizations. The investigated materials were photosensitive copolymers obtained from carbazolylalkylmethacrylates (CEM) and octylmethacrylate (OMA). A detailed investigation was undertaken involving infrared spectroscopy and NMR techniques, ${ }^{1} \mathrm{H},{ }^{13} \mathrm{C}$, COSY, and $\mathrm{HSQC}$, in order to establish the chemical structure and the composition of the copolymers. Holographic recording characteristics were investigated with one- and two-layer photothermoplastic carriers. The two-layer carrier contains separate photosensitive and thermoplastic layers and gives the best holographic response. The surface of microstructured samples has been characterized by atomic force microscopy analysis. It is shown that via a photothermoplastic recording process, it is possible to record and read holograms practically in real time $(\sim 3 \mathrm{~s})$ with a diffraction efficiency of $10 \%$ and a spatial resolution higher than $1000 \mathrm{~mm}^{-1}$.
\end{abstract}

\section{Introduction}

Poly(N-vinylcarbazole) (PVK) has been the subject of intensive investigation in the last 50 years since the discovery of its photoconductivity by Hoegl [1]. In 1957, he established that PVK sensitised with suitable electron acceptors (i.e., 2,4,7-trinitrofluorenone, TNF) showed high enough levels of photoconductivity to be useful in practical applications like electrophotography. Since then, numerous carbazolecontaining polymers have been described in scientific literature and especially as patents [2]. Carbazole-based polymers exhibit good photorefractive, optical and chargetransporting properties [3-5]. In particular, carbazolic compounds have attracted wide interest due to their potential applications in areas such as optical data storage and information processing. In the past decade, special attention was paid to the problem of holographic recording with the aim of protecting the documents from falsification or counterfeiting, and reliefographic methods appeared to be a convenient answer to the problem [6]. Hologram recording in carbazolic polymers can be achieved by several processes using its photorefractive, photoconductive, and/or photochemical properties.

The aim of this work was first to synthesize new carbazolic copolymers appropriated to holographic recording using the photothermoplastic process. For this purpose, the material must have both good photoconductivity and low $T_{g}$. Due to the bulkiness of the carbazolic groups, main chain and side group motions are severely restricted in PVK. The chains are stiff and the polymer has a glass transition temperature around $200^{\circ} \mathrm{C}$ which is among the highest known for vinyl polymers. In addition, PVK is extremely brittle [2]. In order to reduce its $T_{g}$ and to improve its mechanical properties, many polyacrylates and polymethacrylates with pendant carbazole groups have been studied [2]. Polyacrylates in which carbazolic groups are 
TABLE 1: Chemical structure of polymeric units. Protons and carbons are numbered for NMR analysis.

CEM1

separated from the polymer backbone by alkyl spacers of different length have been synthesized by free-radical polymerization. Polymerization leads to materials with moderate molecular weights (Mn) in the range of 5000 to 20000. High molecular weight material $(\mathrm{Mn}=96000)$ is obtained in the case of polymerization of 2-(N-carbazolyl)ethyl acrylate only. An investigation of such polymers by the photoinduced discharge technique has shown that the polyacrylate with the shortest spacer has the highest initial discharge rate. The poly(2-(N-carbazolyl)ethyl acrylate) exhibits charge carrier mobility higher or comparable to that of PVK $[2]$.

In the work presented here, the studied materials were copolymers of 2-(N-carbazolyl)ethyl acrylates (CEM) containing or not octyl methacrylate units (OMA) and synthesized by free-radical polymerization. The role of OMA was mainly to ensure an easy handling of the resulting film without damaging the holographic response of the material. Additionally, it appeared that the presence of OMA in the copolymers with poly(vinylcarbazole) induced a stabilizing effect on the recorded hologram [7]. The three investigated polymeric units are described in Table 1 . The results reported in this paper concern the following formulations: CEM1CEM2 (50-50), CEM1-OMA (60-40), CEM1-OMA (80-20). The numbers in parentheses indicate the mol\% of each component. The first part of this paper describes the copolymers syntheses. With the purpose of firmly assessing the nature of the starting material, the second part presents a detailed characterization of the material that implemented NMR and infrared techniques. The third part of this paper presents holographic investigations with the synthesized copolymers using the photothermoplastic (PTP) recording process (see next section). The experimental investigations were carried out on one- and two-layer PTP carriers. Concerning holographic records with one-layer carriers (polymeric layer simultaneously light sensitive and thermoplastic), the aim was to find the best compromise of composition giving simultaneously suitable rheological properties (adhesiveness, viscosity, low $T_{g}$ ) and good photosensitive properties. Two layers PTP carriers were investigated in order to overcome the restrictions of one layer carriers and to improve the holographic resolving capacity.

Photothermoplastic Process (see [2, 8]). The photothermoplastic (PTP) recording process belongs to methods of electrophotographic optical information recording. A typical PTP organic recording medium (information carrier) is a material having both photosensitivity and thermoplasticity properties. For example, experimentally, a $1-2 \mu \mathrm{m}$ film of carbazolic copolymer of relatively low glass transition temperature, doped with $2 \%-15 \%$ of electron acceptors (i.e., TNF or iodoform), is deposited on a thin layer of metal or oxide $\left(\mathrm{Cr}, \mathrm{In}_{2} \mathrm{O}_{3}\right.$ or $\left.\mathrm{SnO}_{2}\right)$, supported on a transparent substrate (poly(ethylene terephthalate) (PET) or glass). The surface of the polymer is electrostatically charged by a corona discharge in the absence of light. Further holographic exposure leads to the formation of a latent electrostatic image due to a discharging effect and corresponds to the optical image. The electrostatic image is represented by a local distribution of electrostatic forces. Electrostatic contrast of latent image is directly depending on the semiconductor features, that is, photosensibility of the material. For example, carbazole groups and TNF form a charge transfer complex absorbing at $550 \pm 100 \mathrm{~nm}$. The next step consists in developing the image by a thermal treatment. The charges left in unexposed areas are able to deform the photothermoplastic layer when it is heated slightly above its glass transition temperature $\left(T_{g}\right)$. The latent electrostatic image is converted into a relief-phase image, fixed by cooling to room temperature. Information erasure can be achieved by heating the photothermoplastic layer at a temperature higher than its $T_{g}[9,10]$. For the PTP process, in contrast to conventional electrophotographic process, not only photoelectric but also rheological properties of the recording medium are of great importance (i.e., $T_{g}$ ). Instead, no other treatment except the temperature one is necessary.

In the previous example, the copolymer presents simultaneously photosensitivity and thermoplasticity properties and can be used as a one-layer carrier. In a two-layer carrier, the inferior layer is light sensitive and the superior 
one is thermoplastic. The advantage of two layers carriers consists in the possibility of selecting independent materials with required photosensitive and thermoplastic properties. These materials have been recognized as being promising for reversible holographic data storage allowing holographic recording, reading, and erasing.

\section{Experimental}

2.1. Materials. Carbazole and poly(vinylcarbazole) (PVK) powders containing no stabilisers or additives were obtained from Aldrich. Ethylene oxide, n-octanol, AIBN (2,2'azobisisobutyronitrile), benzoyl chloride, and methacrylic acid were of the highest available quality (Aldrich) and used as received. Octylmethacrylate was prepared by reaction of $n$-octanol on methacryloyl chloride.

2.2. NMR Experiments. ${ }^{1} \mathrm{H}$ and ${ }^{13} \mathrm{C}$ NMR spectra were recorded with both 300 and 500 Avance Bruker spectrometers, (with or without gradient-probes, depending on the used program, noted ge- in the case of using gradients), at $23^{\circ} \mathrm{C}$, on the products in $\mathrm{CDCl}_{3}$ solution (taken as an internal reference at 7.28 and $77.3 \mathrm{ppm}$ ). All resonances were unambiguously assigned using homonuclear $\mathrm{H}-\mathrm{H}$ as well as heteronuclear one-bond and long-range ${ }^{1} \mathrm{H}_{-}{ }^{13} \mathrm{C}$ correlations extracted from ge-COSY (Correlation Spectroscopy), geHSQC (Heteronuclear Single Quantum Coherence), and geHSQC-lr (optimised for long-range correlation).

2.3. Infrared Experiments. Before infrared analysis, samples were prepared by evaporation from a polymer dichloromethane solution on $\mathrm{KBr}$ plates at room temperature. The deposits were then dried in vacuum at room temperature. The solvent evaporation was carefully controlled by IR analysis. Infrared spectra of the samples were recorded with a Nicolet Magna-IR 860 ( $4 \mathrm{~cm}^{-1}$ and 32 scans).

2.4. SEC and DSC Measurements. Macromolecular weights were measured by Size Exclusion Chromatography with a Viscotek GMHXl column (length $=30 \mathrm{~cm}$, stationary phase $=\mathrm{PSDVB}$, temperature $=30^{\circ} \mathrm{C}$, flow rate $=1 \mathrm{~mL} \cdot \mathrm{min}^{-1}$ ) and a Viscotek VE 3580 viscosimetry detector, in tetrahydrofuran solution. The Differential Scanning Calorimeter (DSC) used to measure the glass transition $T_{g}$ was a Mettler Toledo DSC30.

2.5. Holographic Setup. To record holograms on the PTP media, an installation with an Nd:YAG DPSS green laser (1) from Laser-compact Group ( $\lambda=532 \mathrm{~nm}$, model LCMS-111, coherence length $>50 \mathrm{~m}$, output power $50 \mathrm{~mW}$ ) was mounted (Figure 1). Two laser beams of equal radiation intensity obtained by a cubic beam splitter (5) reunite at the angle of $2 \theta$, forming the interference grating on the surface of sample (8). The spatial frequency of grating is determined by laser wavelength $\lambda$ and angle $\theta$ in accordance with the optic lows. To read the recorded hologram, a nonenlarged beam (11) of an He-Ne laser $(\lambda=633 \mathrm{~nm})$ is used. The role of the PTP process control unit (9) is to carry out the operations

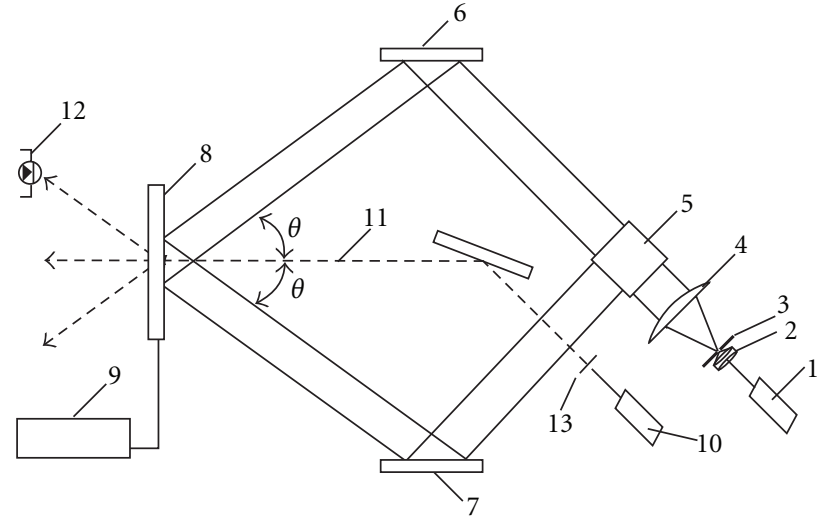

FIGURE 1: Holographic setup. 1-DPSS laser $\lambda=532 \mathrm{~nm}, 2-$ micro-objective, 3-pinhole, 4-collimator, 5-cubic beam splitter, 6,7mirrors, 8-sample, 9-recording process control unit, 10-He Ne laser $\lambda=0.633 \mu \mathrm{m}, 11$-read-out beam, 12-photodetector.

of recording and thermal development of holograms in an automatic mode. The diffraction efficiency is measured after complete process. It is defined as the ratio of light intensity at the first diffraction order and of the incident signal (light absorption in the PTP carrier is considered).

2.6. Atomic Force Microscopy Measurements. Images acquisition was performed by Atomic Force Microscopy (AFM), in Tapping Mode, on a Nanoscope IIIa microscope from Veeco. Topography images were recorded with a tapping silicon tip (RTESP-NCL, Veeco) with approximate values of 150 $250 \mathrm{kHz}$ resonant frequency, $20-70 \mathrm{~N} \cdot \mathrm{m}^{-1}$ spring constant, and $5-10 \mathrm{~nm}$ radius of curvature.

\section{Results and Discussion}

3.1. Synthesis of the Monomers. M1 and M2 monomers synthesis (corresponding to CEM1 and CEM2, respectively, in the polymeric structure) was carried out as described elsewhere [11] (Figure 2). The condensation of ethylene oxide with carbazole (methylethylketone as solvent) involved in the process gave rise to a rather complex mixture of oligomers, from which the desired products were isolated by liquid chromatography (silica gel, chloroform as eluent). The first eluted product corresponded to the monocondensation (Figure $1, n=0, \mathrm{mp}=83^{\circ} \mathrm{C}$ ) and was isolated, with a moderate yield ( $\mathrm{ca} 50 \%$, based on the carbazole consumption). The heavier ether-alcohols $(n=1,2, \ldots)$ were then eluted in the order of increasing mass. The second step was straightforward and consisted in the reaction between the corresponding alcohol and methacryloyl chloride, in the presence of triethylamine. The acyl halide was freshly prepared by reaction of methacrylic acid on benzoyl chloride and directly distilled in toluene solution of the alcohol. After classical workup, the crude mixture was chromatographed (silica gel, chloroform as eluent) to afford M1 and M2 which were obtained, in this order, as solids (yield $\approx 52$ and $30 \%$, resp.). The purity of the monomers was monitored by thin 
TABLE 2: ${ }^{1} \mathrm{H}$ and ${ }^{13} \mathrm{C}$ chemical shifts of the olefinic monomers (M1 and M2) and of their homologous structures (CEM1 and CEM2 together with OMA) in the macromolecules after polymerization. The numbers/letters in the first column correspond to the atoms numbering as indicated in Table 1.

\begin{tabular}{|c|c|c|c|c|c|c|}
\hline & \multicolumn{2}{|c|}{ M1 } & \multicolumn{2}{|c|}{ M2 } & \multicolumn{2}{|c|}{ Copolymers } \\
\hline & ${ }^{1} \mathrm{H}$ & ${ }^{13} \mathrm{C}$ & ${ }^{1} \mathrm{H}$ & ${ }^{13} \mathrm{C}$ & ${ }^{1} \mathrm{H}$ & ${ }^{13} \mathrm{C}$ \\
\hline 1 & $5.97,5.51$ & 126.30 & $6.08,5.59$ & 128.43 & $1.5-2.0$ & $52-57$ \\
\hline 2 & & 135.63 & & 135.96 & & 44.8 \\
\hline $\mathrm{CH}_{3}$ & 1.84 & 18.17 & 1.96 & 18.21 & $0.2-1.3$ & 17,19 \\
\hline 3 & & 167.22 & & 167.24 & & $176-178$ \\
\hline $4 a$ & 4.57 & 62.44 & & & $4.0-4.4$ & 62.5 \\
\hline $4 \mathrm{~b}$ & & & 4.26 & 63.61 & $3.7-3.9$ & 63.8 \\
\hline $4 c$ & & & & & $3.7-3.9$ & 65.3 \\
\hline $5 a$ & 4.62 & 41.55 & & & $4.2-4.7$ & 41 \\
\hline $5 b$ & & & 3.66 & 69.2 & $3.2-3.5$ & 68.7 \\
\hline $5 c$ & & & & & $2.4-2.7$ & 28.1 \\
\hline $6 b$ & & & 4.55 & 69.41 & $3.5-3.9$ & 69.2 \\
\hline $6 c$ & & & & & $1.15-1.3$ & 26 \\
\hline $7 b$ & & & 3.95 & 43.16 & $4.2-4.5$ & 42.9 \\
\hline $7 \mathrm{c}$ & & & & & $1.15-1.3$ & 29.2 \\
\hline 8 & & & & & $1.15-1.3$ & 29.2 \\
\hline 9 & & & & & $1.15-1.3$ & 31.75 \\
\hline 10 & & & & & $1.15-1.3$ & 22.6 \\
\hline 11 & & & & & $0.8-0.9$ & 14.1 \\
\hline $\mathrm{a}$ & & 140.36 & & 140.54 & & 140.2 \\
\hline $\mathrm{b}$ & 7.48 & 108.57 & 7.52 & 108.78 & $7.3-7.4$ & 108.6 \\
\hline c & 7.51 & 125.74 & 7.52 & 125.63 & $7.3-7.4$ & 125.7 \\
\hline d & 7.26 & 119.22 & 7.31 & 118.99 & 7.2 & 119.3 \\
\hline e & 8.14 & 120.35 & 8.16 & 120.22 & 8 & 120.4 \\
\hline $\mathrm{f}$ & & 123.00 & & 122.88 & & 122.9 \\
\hline
\end{tabular}

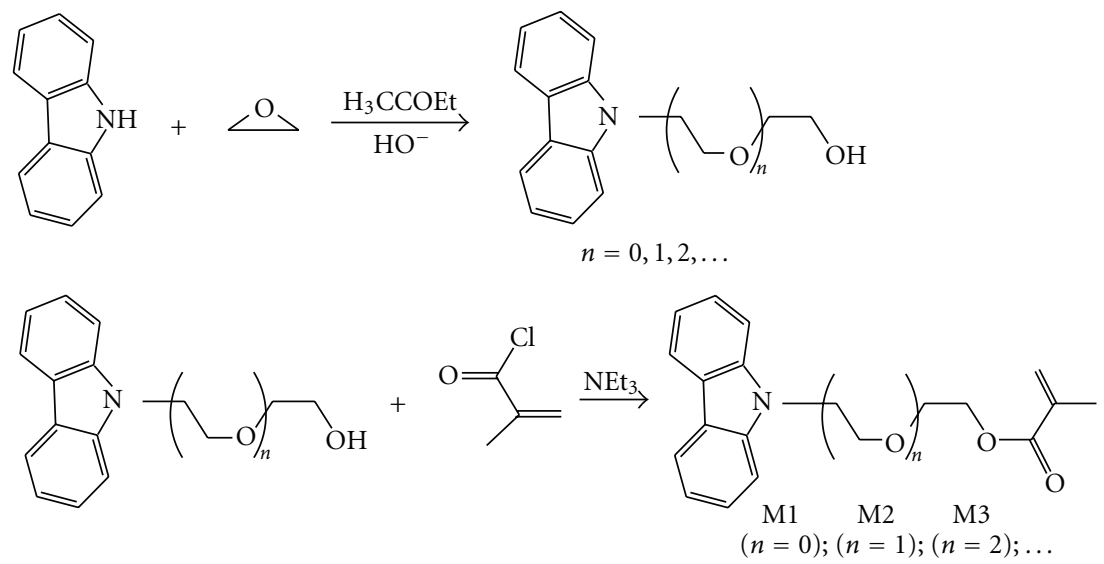

FIgURE 2: Synthesis of the monomers bearing the carbazole nucleus.

layer chromatography and NMR. For instance, HSQC of M1 and COSY spectra of M2 are shown in Figures 3 and 4. On HSQC spectrum of M1 (Figure 3), the couple of aliphatic protons from $\mathrm{CH}_{2}$ shows an A2B2 second-order spectrum with very close chemical shifts (around 4.57 and 4.62, for protons $4 \mathrm{a}$ and 5a, resp.; see Tables 1 and 2).
These protons appear to be clearly correlated with carbons at 62.44 and $41.55 \mathrm{ppm}$ whereas in the case of the higher homologue $\mathrm{M} 2$, resonances of the pendant chain nucleus are significantly different. The COSY spectrum of M2 (Figure 4) gives evidence for two correlations, between $4 \mathrm{~b}$ and $5 \mathrm{~b}$ at 4.26 and $3.66 \mathrm{ppm}$ on one hand, and $6 \mathrm{~b}$ and $7 \mathrm{~b}$ at 4.55 


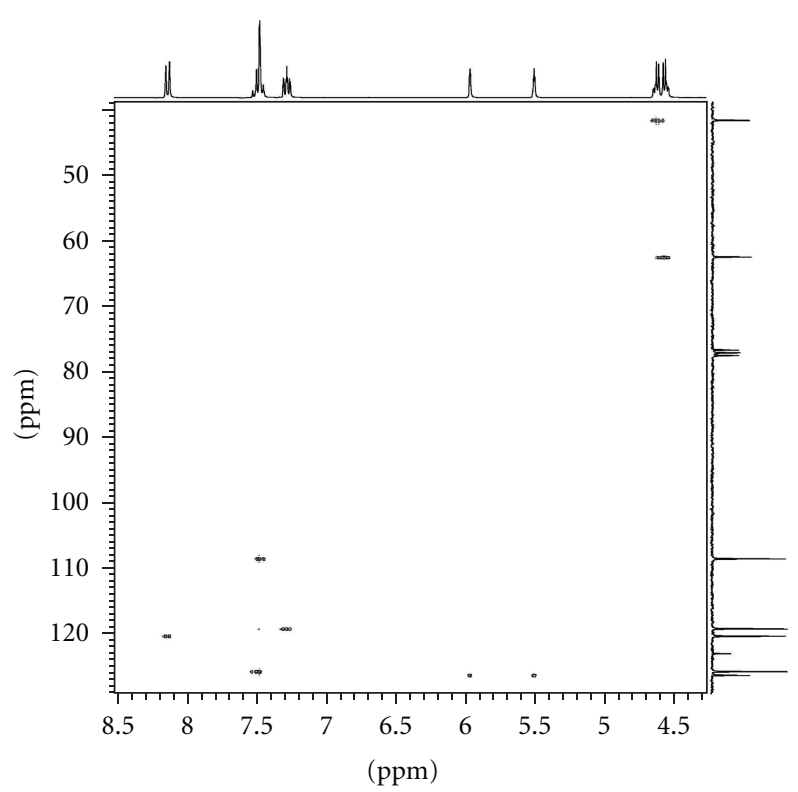

FIgURE 3: HSQC spectrum of the monomer M1.

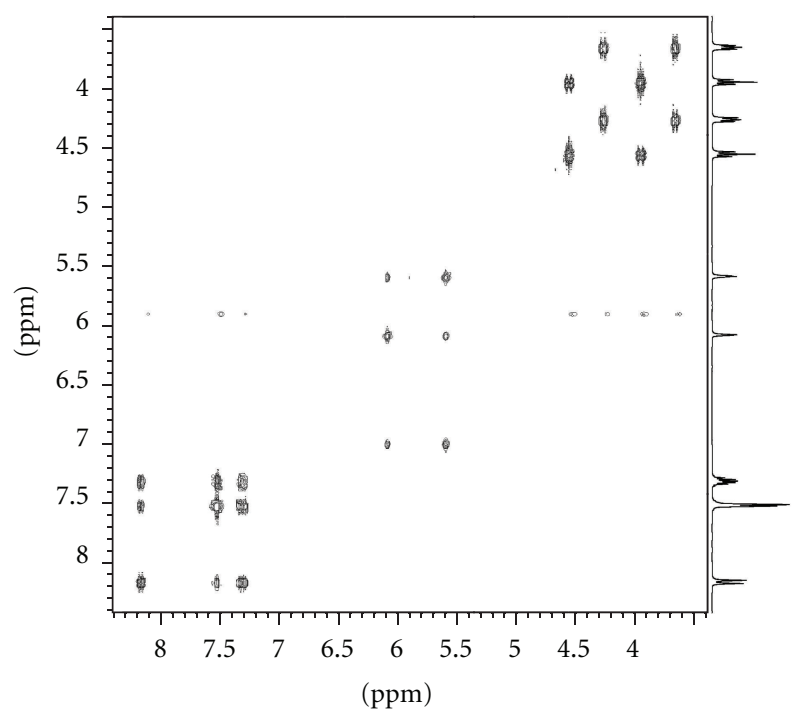

Figure 4: COSY spectrum of the monomer M2.

and $3.95 \mathrm{ppm}$ on the other hand (cf., formula in Table 1 for proton and carbon numbering).

This detailed analysis of the monomers was of indispensable interest to further attribute all the resonances in the corresponding polymers: each macromolecule can be fully characterised by a carbon resonance of a precise monomer. Accordingly, it is then possible to determine the copolymer composition and to check the agreement or not between the expected composition from the stoichiometry used in the synthesis and the so obtained values.

3.2. Polymerization. Copolymers used in this work (Table 1) were prepared by radical polymerization (AIBN as an initiator, toluene solution, $85^{\circ} \mathrm{C}$ ) as already described [11].
The purification was carried out by precipitation (at least twice) from a toluene solution of the crude product into a wide excess of methanol. The polymers were filtrated, washed with methanol, and dried under vacuum in the dark. The purity and the composition of the copolymers were then checked by NMR and IR spectroscopy (cf, later). Average molecular weight $\mathrm{Mw}$ of $110000 \mathrm{~g} \cdot \mathrm{mol}^{-1}$ for CEM1-CEM2 and CEM1-OMA was obtained.

3.3. NMR Initial Characterization. Assignment of most of the signals in NMR spectra of polymer samples was based on the ${ }^{1} \mathrm{H}_{-}{ }^{13} \mathrm{C}$ correlation extracted from HSQC spectra and the ${ }^{1} \mathrm{H}$ and ${ }^{13} \mathrm{C}$ chemical shifts of monomers. In copolymer spectra, only signals belonging to protons and carbons of the main chain exhibit some fine structure (however, not resolved) that can be related to structural variation of the macromolecule. Signals belonging to the side chains (although sometimes rather broad) are not sensitive to the structural variation of the copolymer because of the large distance of these fragments from knots of the structural variation.

From the fragments located on the main chain or in its close vicinity, the $\mathrm{CH}_{3}$ group exhibits two well-resolved peaks in the carbon spectra and unusual broad proton resonance. $\mathrm{CH}_{2}$ group provides broad and not resolved signals in both proton and carbon spectra. Among the two quaternary carbons, the carbon of the main chain presents a relatively narrow signal, while $\mathrm{C}^{3}$ carbon signal is rather broad, poorly resolved into two parts. The shape of all these signals is not affected by the variation of monomer composition in the copolymer. It implies that the observed fine structure of the main chain resonances must be due to the stereochemical isomerism, for example, due to different tacticity. Copolymers have block character or the NMR spectra are insensitive to the regio-isomerism.

Assignments of proton and carbon spectra of polymers are shown in Tables 1 and 2 (full detailed data on request). The monomer data $\left({ }^{1} \mathrm{H},{ }^{13} \mathrm{C}\right.$ of $\mathrm{M} 1$ and $\left.\mathrm{M} 2\right)$ at the origin of the assignment of all the resonances in the copolymers are also given.

As an example, the results related to the copolymer CEM1-OMA (60-40) are detailed in this part. As usual for organic polymers, proton NMR spectrum is not very informative. Subspectra of aromatic and of $\mathrm{CH}_{2}-\mathrm{CH}_{2}$ proton system exhibited high-order feature, so only approximate chemical shifts may be determined. On the contrary, and considering the appearance of the aliphatic region of the ${ }^{13} \mathrm{C}$ carbon spectra (Figure 5), it was obvious that there were two clearly split peak positions in the $\alpha-\mathrm{OCH}_{2}$ resonance area, situated at 62.5 (4a) and $65.3 \mathrm{ppm}(4 \mathrm{c})$ and corresponding to CEM1 and OMA, respectively. Integral measurements (quantitative sequence as well as $30^{\circ}$ pulses and long relaxation delays were used) directly yielded the ratio of the two monomers, 61-39 versus 60-40 (theor.) in that case. The composition can also be checked in the upfield region of the spectrum. Two rather broad resonances centred at $18 \mathrm{ppm}\left(\mathrm{CH}_{3}\right.$ of the main chain) appeared beside a narrow peak at $14.1 \mathrm{ppm}$ belonging to the terminal methyl of the octyl residue. Under our standard conditions 


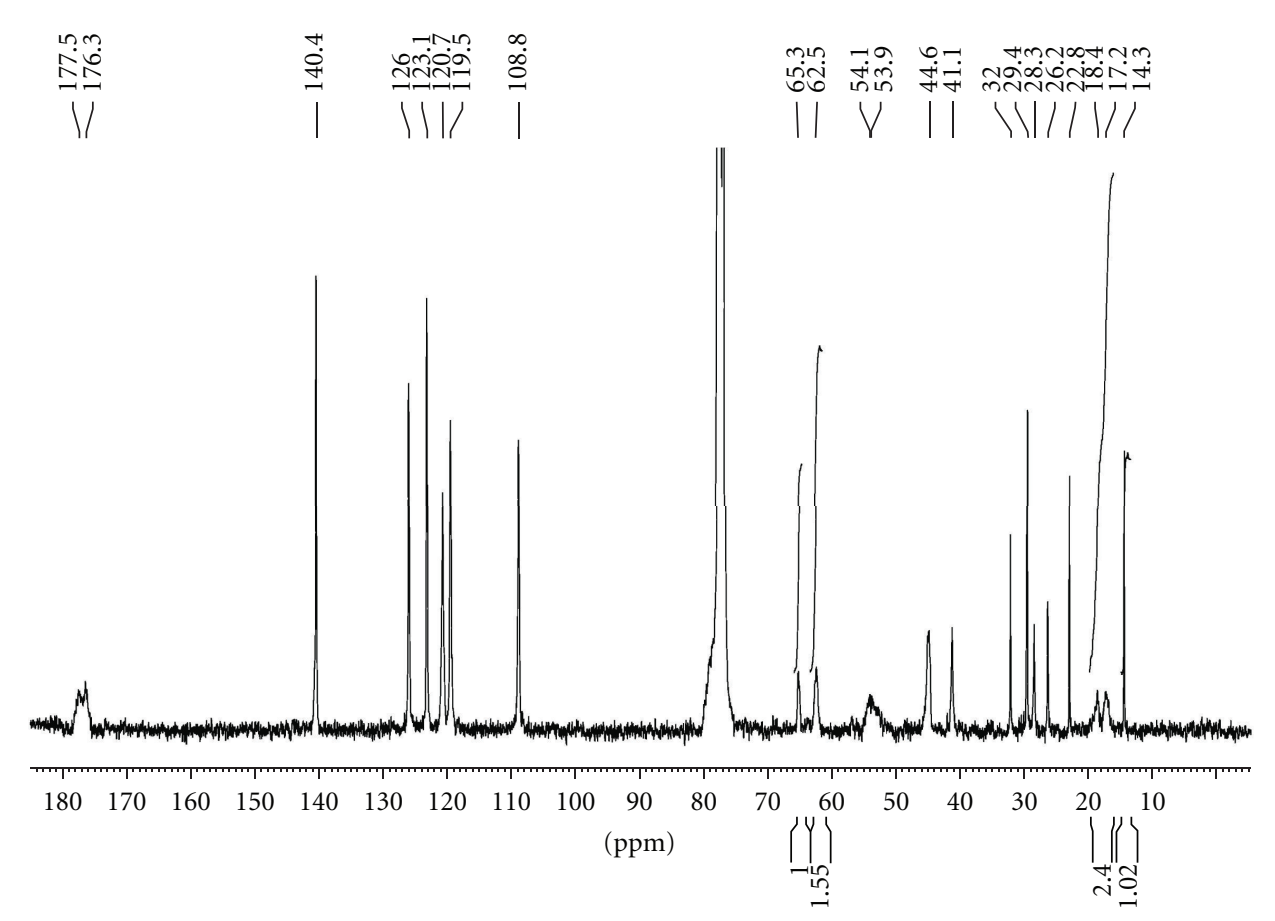

FIgURE 5: ${ }^{13} \mathrm{C}$ spectrum of CEM1-OMA (60-40) recorded with inverted-gated "quantitative" sequence.

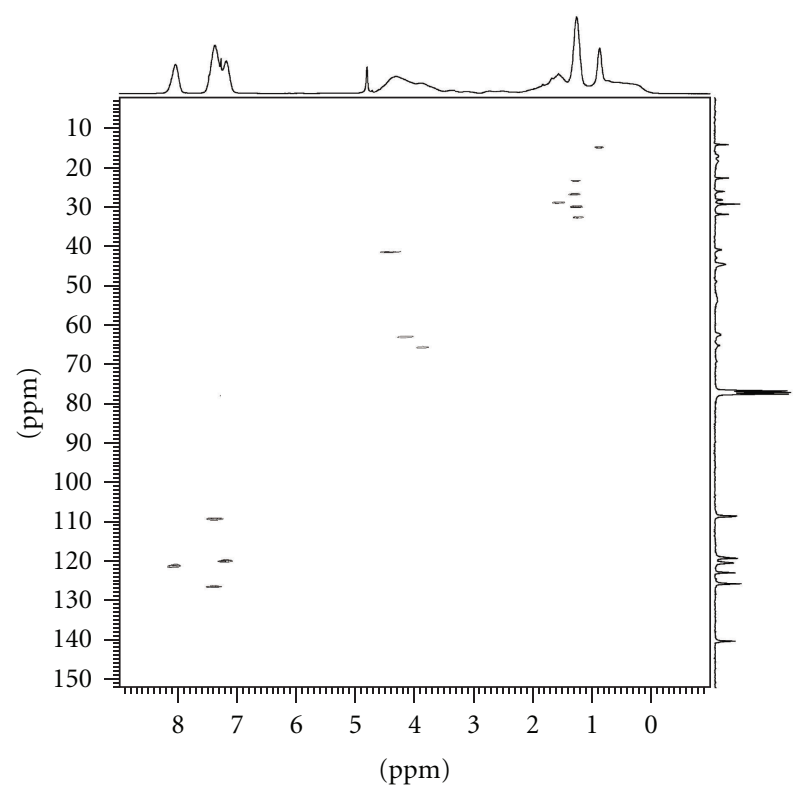

FIGURE 6: HSQC spectra of CEM1-OMA (60-40).

$(300 \mathrm{MHz})$, the carbazole moieties of the two monomers are undistinguishable. The result was further confirmed with HSQC spectrum and the observed heteronuclear correlation (Figure 6).

The copolymer CEM1-CEM2 (50-50) comprising side groups with a longer chain was also investigated by following the same procedure: the composition was confirmed from the analysis of the quantitative ${ }^{13} \mathrm{C}$ spectrum based on peaks of each monomer, at $62.6 \mathrm{ppm}$ (carbon 4a) for CEM1 and at
68.6 and $69.2 \mathrm{ppm}$ (carbon $5 \mathrm{~b}$ and/or 6b, resp.) for CEM2 (Figure 7). The results were in good agreement with the expected values again.

3.4. Infrared Characterization. The copolymers were also investigated by infrared spectroscopy. The spectra of the three copolymers have been compared to the spectra of commercially available PVK and of PMMA (poly(methyl methacrylate)) [12]. PVK and PMMA were used as references for attributions of absorption bands of, respectively, carbazole and OMA groups. Spectra of PVK, CEM1:CEM2, and CEM1:OMA are given in Figure 8. Attributions of infrared absorption bands are reported in Table 3. Each organic group has been identified on the basis of literature data [13]. The obtained data confirmed the chemical structure of copolymers. In addition, we quantitatively compared the composition of copolymers. This composition was indirectly evidenced determining the carbazole content (in wt\%). The absorbance of the carbazolic band at $1597 \mathrm{~cm}^{-1}$ (ring stretch) has been measured for copolymers and PVK films of the same thickness $(15 \mu \mathrm{m})$. The results show a good agreement between the infrared measurements and the expected values (within $4 \%$, Table 4 ).

3.5. Holographic Recording. The experiments were carried out on one- and two-layer PTP carriers. First of all, several copolymer compositions were tested varying from 50 to $80 \mathrm{~mol} \%$ of CEM1 units. One-layer carriers were obtained by evaporation of a copolymer solution cast on a PET film covered by a transparent chrome electrode (thickness $0.1 \mu \mathrm{m}$ ). It has been observed that when the percentage of CEM1 units increases, the photosensitive properties increase 


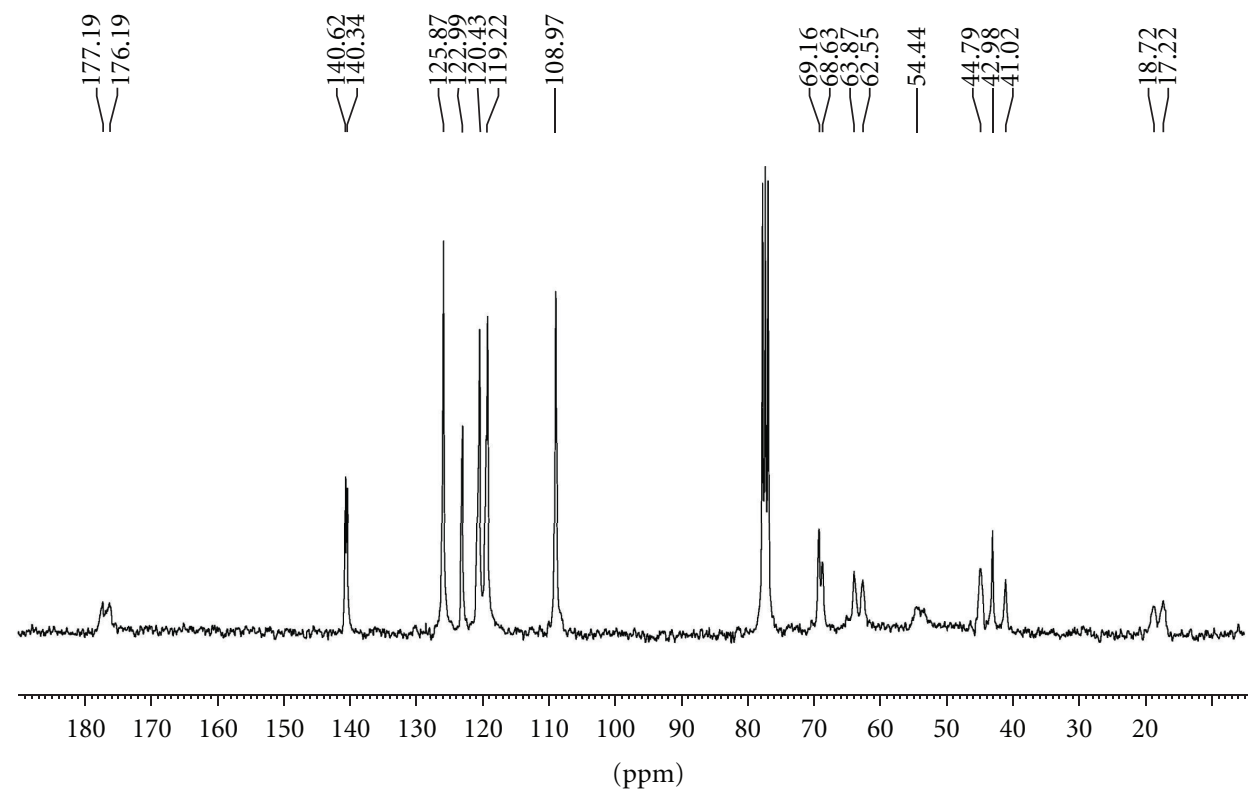

Figure 7: ${ }^{13} \mathrm{C}$ spectrum of CEM1-CEM2 (50-50).
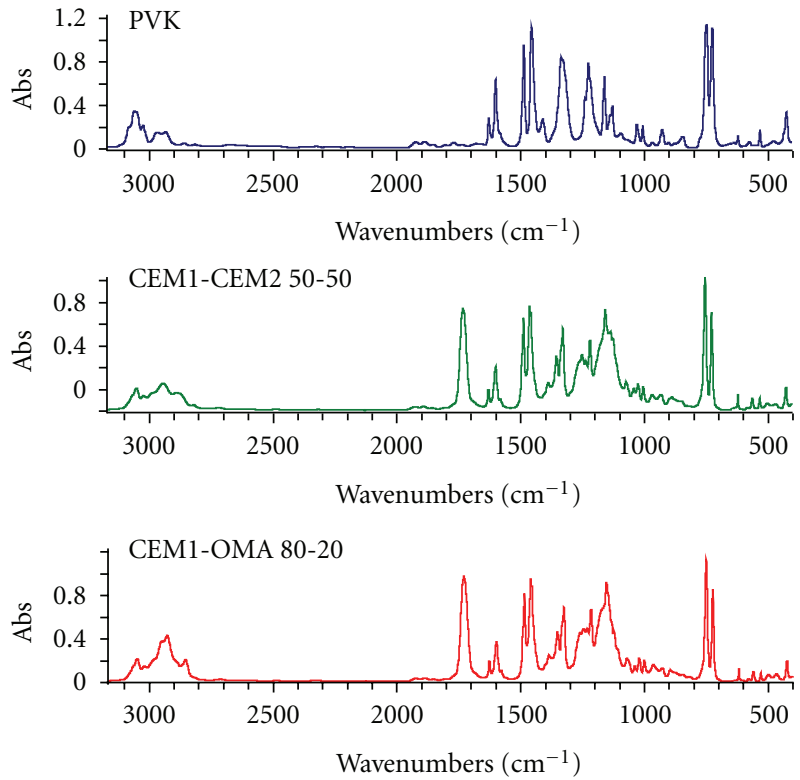

Figure 8: Infrared spectra of PVK, CEM1-CEM2 (50-50) and CEM1-OMA (80-20) (films thickness $15 \mu \mathrm{m}$ ).

and the rheological properties decrease. The best holographic results were obtained with the photopolymer CEM1OMA $(60-40)+12 \mathrm{wt} \%$ of TNF. Specimens with different copolymer thickness $(1.8 \mu \mathrm{m}, 1.6 \mu \mathrm{m}, 1.4 \mu \mathrm{m})$ were prepared. On the holographic setup, the optimal recording parameters of specimens were determined-warming-up temperature to achieve the plasticity state of polymer $\left(T_{g}=83^{\circ} \mathrm{C}\right)$, potential of corona discharge $(9.5 \mathrm{kV})$, and recording time $(\approx 3 \mathrm{~s})$. Dependence of diffraction efficiency on recording spatial frequency (lines per millimetres) for carriers with different

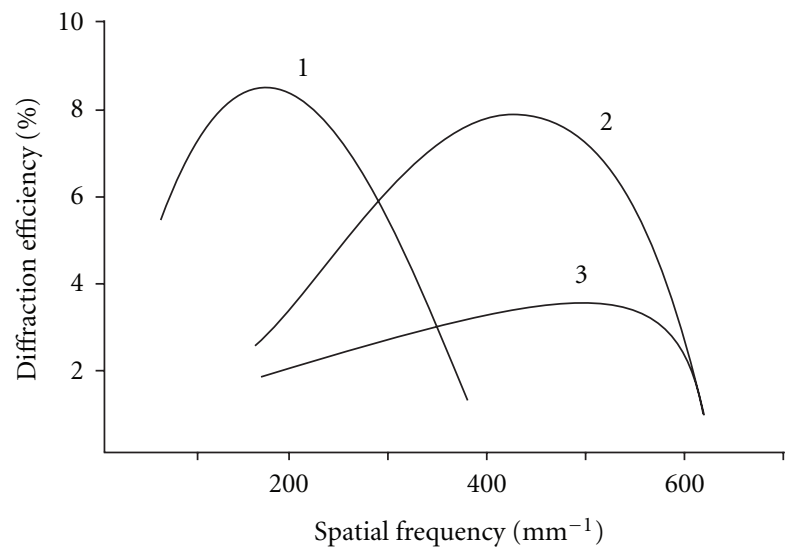

FIGURE 9: Diffraction efficiency versus spatial frequency for one layer carriers with different thickness of the PTP layer: (1) $1.8 \mu \mathrm{m}$, (2) $1.6 \mu \mathrm{m}$, (3) $1.4 \mu \mathrm{m}$.

photopolymer thickness is presented in Figure 9. With a layer thickness of $1.8 \mu \mathrm{m}$ (Figure 9, curve 1), a maximal value of diffraction efficiency $\eta=8.5 \%$ is achieved at the spatial frequency of $185 \mathrm{~mm}^{-1}$. Further increase of frequency leads to a decrease of diffraction efficiency till $1 \%$ at the spatial frequency $420 \mathrm{~mm}^{-1}$. To record holograms with higher spatial frequencies, the thickness of the layer should be reduced. However, a decrease of the thickness layer also leads to a decrease of the maximal diffraction efficiency value $(\eta=7.5 \%$ is achieved at the spatial frequency of $420 \mathrm{~mm}^{-1}$ for a layer thickness of $1.6 \mu \mathrm{m}$, Figure 9, curve 2). A further decrease of thickness $(1.4 \mu \mathrm{m}$, Figure 9, curve 3) leads to a quick decay in diffraction efficiency with a maximal spatial frequency of $620 \mathrm{~mm}^{-1}$. The surface of the samples has been characterised by AFM (Figure 10). 
TABLE 3: Attribution of infrared absorption bands.

\begin{tabular}{|c|c|c|c|c|}
\hline PVK & CEM1-CEM2 (50-50) & CEM1-OMA (60-40 or $80-20)$ & PMMA & Attribution \\
\hline & 3484 & 3484 & 3439 & $\mathrm{C}=\mathrm{O}$ stretch overtone (ester) \\
\hline 3081 & 3081 & 3081 & & \\
\hline 3049 & 3049 & 3049 & & Aromatic C-H stretch \\
\hline \multirow[t]{2}{*}{3022} & 3022 & 3022 & & \\
\hline & 2986 & 2986 & 2993 & $\mathrm{CH}_{3}$ antisymetric stretch \\
\hline \multirow{3}{*}{2964} & \multirow{2}{*}{2941} & 2953 & 2953 & $\mathrm{CH}_{3}$ antisymetric stretch \\
\hline & & 2928 & 2922 & $\mathrm{CH}_{2}$ antisymetric stretch \\
\hline & \multirow{2}{*}{2878} & 2880 and 2869 & 2880 & $\mathrm{CH}_{3}$ symetric stretch \\
\hline \multirow[t]{2}{*}{2856} & & 2854 & 2843 & $\mathrm{CH}_{2}$ symetric stretch \\
\hline & 1728 & 1728 & 1730 & $\mathrm{C}=\mathrm{O}$ stretch \\
\hline 1625 & 1628 & 1628 & & \\
\hline 1597 & 1597 & 1597 & & Ring stretch \\
\hline \multirow[t]{3}{*}{1483} & 1485 & 1485 & & \\
\hline & 1460 & 1460 & & $\mathrm{CH}_{3}$ antisymetric bend \\
\hline & & & 1445 & "scissors" $\mathrm{CH}_{2}$ bend \\
\hline \multirow[t]{3}{*}{1452} & 1454 & 1454 & & "scissors" $\mathrm{CH}_{2}$ bend overlapped with "sideways" ring stretch \\
\hline & 1385 & 1385 & 1388 & $\mathrm{CH}_{3}$ "umbrella" symmetric bend \\
\hline & 1351 & 1351 & & $\mathrm{CH}_{2}$ wagging bend (pendant groups) \\
\hline 1332 & 1332 & 1332 & & $\mathrm{C}_{\text {aromatic }}-\mathrm{N}$ stretch \\
\hline \multirow[t]{3}{*}{1325} & 1325 & 1325 & & $\mathrm{CH}$ bend \\
\hline & 1260 & 1260 & 1268 & \\
\hline & 1247 & 1247 & 1242 & $\mathrm{C}(\mathrm{O})$-O-C stretch \\
\hline 1236 & 1230 & 1230 & & $\mathrm{C}_{\text {carbazole }}-\mathrm{N}$ stretch \\
\hline \multirow[t]{2}{*}{1224} & 1215 & 1215 & & $\mathrm{C}_{\text {aliphatic }}-\mathrm{N}$ stretch \\
\hline & 1160 (broad) & 1160 (broad) & & $\mathrm{O}-\mathrm{C}(\mathrm{O})-\mathrm{C}$ stretch \\
\hline 1158 & 1153 & 1153 & 1149 & $\mathrm{C}-\mathrm{C}$ main chain or $\mathrm{CH}_{2}$ rocking \\
\hline 1132 & 1132 & 1132 & & carbazole \\
\hline 1122 & 1122 & 1122 & & carbazole \\
\hline 1027 & 1021 & 1021 & & Ortho-substituted aromatic cycle \\
\hline 745 & 751 & 751 & 752 & Out-of-plane bending of aromatic C-H \\
\hline 721 & 724 & 724 & & Concerted rocking of the $\mathrm{CH}_{2}$ \\
\hline
\end{tabular}

TABLE 4: Infrared quantification of carbazole groups.

\begin{tabular}{lccccc}
\hline Material & Mol\% & Wt $\%$ & Wt $\%$ of carbazole groups (cal.) & Abs at $1597 \mathrm{~cm}^{-1}(15 \mu \mathrm{m})$ & Wt $\%$ of carbazole groups (exp.) \\
\hline PVK & & & 86 & 0.608 & 52 \\
CEM1-CEM2 & $50-50$ & $46-54$ & 55 & 0.364 & 38 \\
CEM1-OMA & $60-40$ & $68-32$ & 40 & 0.267 & 47 \\
CEM1-OMA & $80-20$ & $85-15$ & 51 & 0.334 & 47 \\
\hline
\end{tabular}

As expected, after recording and developing the image by thermal treatment, the three-dimensional plot shows the formation of a surface relief grating. As recalled in the introduction, the charges left in unexposed areas are able to deform the thermoplastic layer when it is heated slightly above its glass transition temperature $\left(T_{g}\right)$. During heating, the remaining positive charges localized at the surface attempt to get closer to the negative charges underneath; a deformation of the surface occurs, creating depressions in the relief. Those depressions are clearly observed in the AFM image (Figure 10(a)). The relief profile (Figure 10(b)) shows the same spatial periodicity $(\sim 5 \mu \mathrm{m})$ as the light pattern spacing. The corrugations height varies from 0.8 to $1.8 \mu \mathrm{m}$. The thickness of the photothermoplastic layer of this sample was $1.8 \mu \mathrm{m}$, so this result indicates that the deformation occurs in the whole thickness of the layer. After development, the imprint of the charges remains in the sample. This phenomenon is illustrated in Figure 11. This image is a top view of the surface; a scale in height with level lines has been intentionally chosen. The dark strips represent the top 


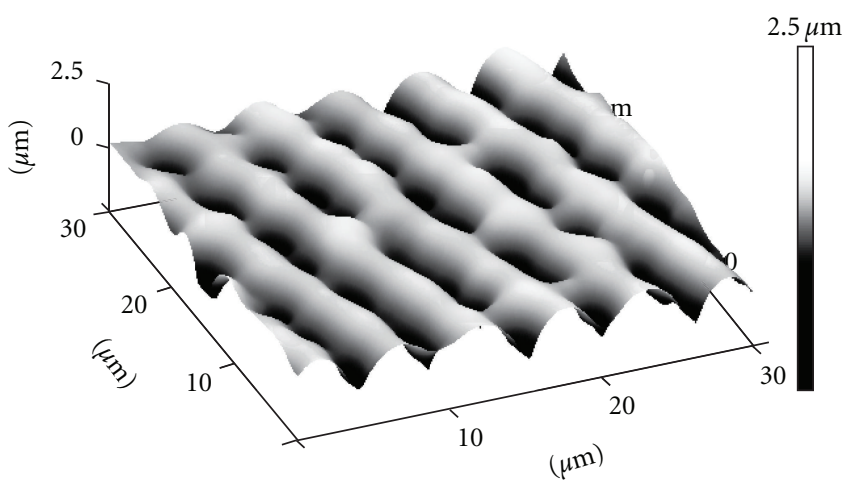

(a)

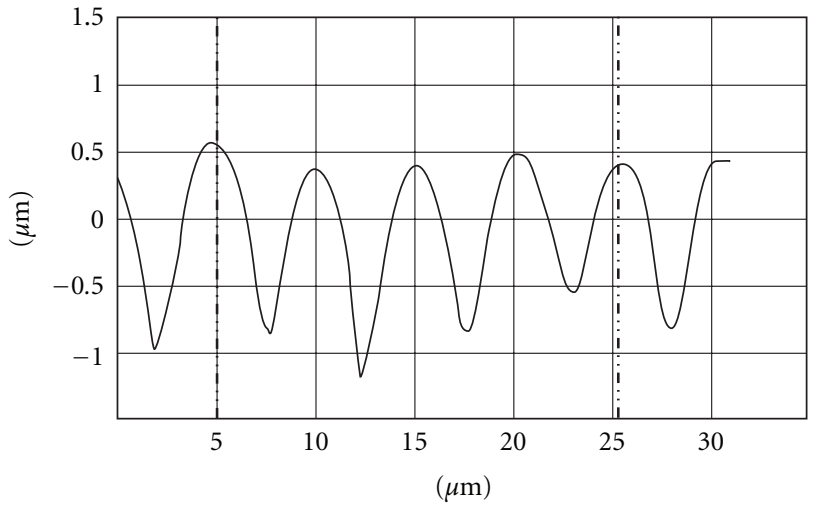

(b)

FIGURE 10: AFM analysis of the surface of a one-layer PTP carrier after holographic recording and thermal developing; the thickness of the photopolymer layer is $1.8 \mu \mathrm{m}$. (a) 3D surface plot, (b) relief profile.

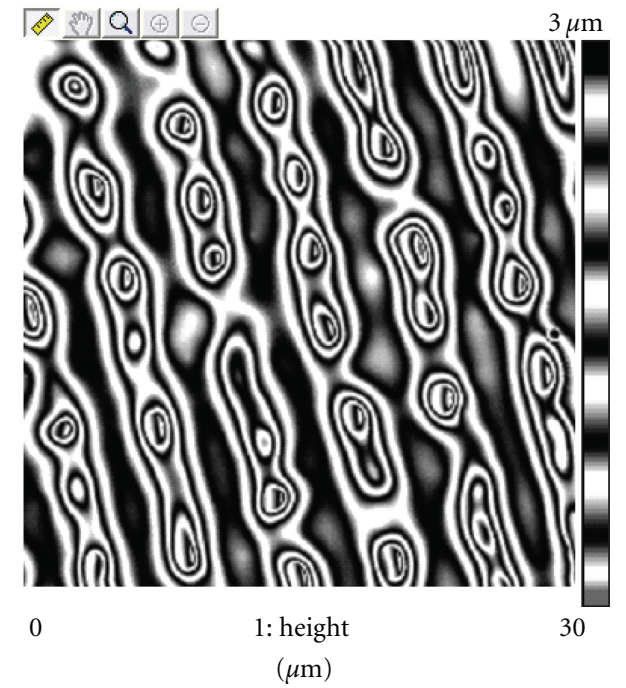

Figure 11: AFM analysis, top view of the same image as the one represented in Figure 10(a), the scale in height is represented by level lines.

of the corrugations and the pseudocircles are located in the depressions of the relief and correspond to the places where the highest concentrations of charges were localized.

Even if interesting features were evidenced by AFM analysis, the holographic response of the one-layer carrier is limited. In order to improve the holographic characteristics, a study of two layers carriers was carried out. In this case, it is possible to freely vary the electrical and geometrical parameters of each functional level independently. Two-layer carriers were obtained by deposition of a photopolymer layer (thickness $1.8 \mu \mathrm{m}$ ) on a metal electrode. A thermoplastic top layer (thickness $0.5 \mu \mathrm{m}$ ) was then deposited on the photopolymer layer. The best results were obtained with a photopolymer layer of CEM1:OMA $\left(60-40+12 \mathrm{wt} \%\right.$ of TNF, $\left.T_{g}=83^{\circ} \mathrm{C}\right)$ and a thermoplastic layer of styrene-butylmethacrylate copolymer $\left(50-50, T_{g}=54-55^{\circ} \mathrm{C}\right)$. In this case, the plot

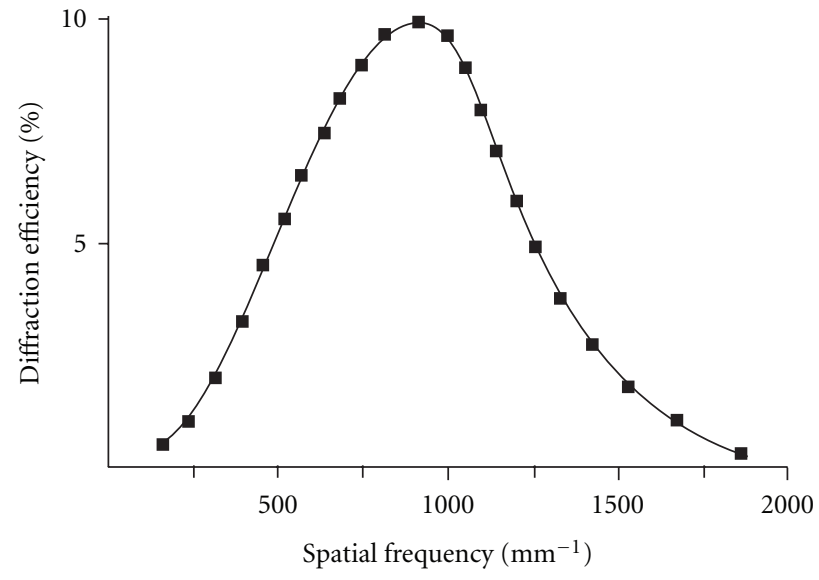

FIGURE 12: Diffraction efficiency versus spatial frequency for a twolayer carrier with a thickness of PTP layer of $1.8 \mu \mathrm{m}$ and a thickness of thermoplastic top layer of $0.5 \mu \mathrm{m}$.

of diffraction efficiency versus spatial frequency (Figure 12) shows that a maximal value of diffraction efficiency of $\eta=$ $10 \%$ is obtained at a spatial frequency of about $1000 \mathrm{~mm}^{-1}$. In comparison with the one-layer carrier (Figure 9), the resolving capacity of the two-layer carriers is interestingly increased and the spatial frequency reaches $1800 \mathrm{~mm}^{-1}$ (with $\eta=0.1 \%)$. This better resolution is explained by the lower temperature used for the thermal treatment $\left(58^{\circ} \mathrm{C}\right.$ instead of $83^{\circ} \mathrm{C}$ ). Dark conductivity of semiconductors sharply increases with increasing temperature and correspondingly, sharply decreases the photosensibility of material $[14,15]$. In the case of the one-layer carrier, the temperature leads to a degradation of the latent electrostatic image and to a limitation of the carrier's capacity of resolution (the best spatial frequency obtained was $620 \mathrm{~mm}^{-1}$ ). In the two-layer carrier, charges leakage from light regions to dark ones is reduced and better spatial resolution has been achieved $\left(1800 \mathrm{~mm}^{-1}\right)$. AFM surface analysis (Figure 13) confirmed 


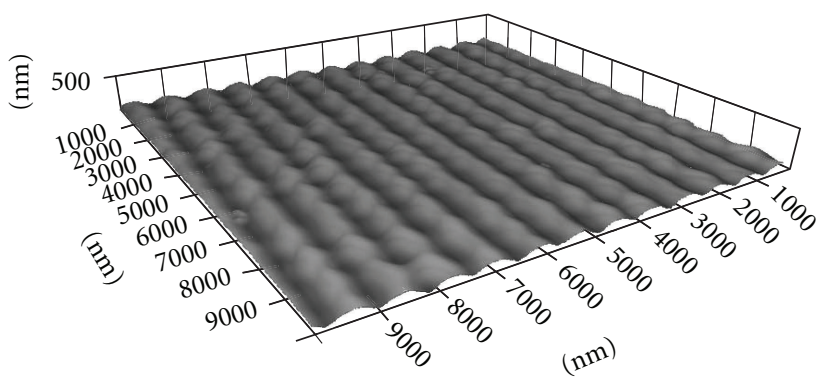

(a)

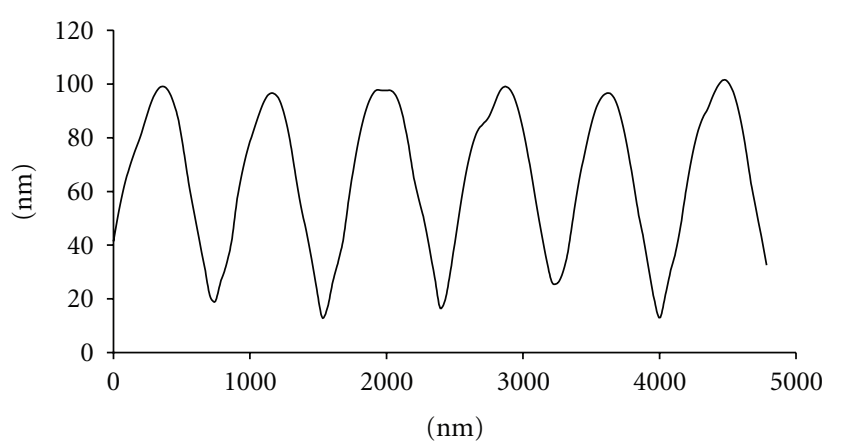

(b)

FIGURE 13: AFM analysis of a two-layer PTP carrier after holographic recording and thermal developing; thicknesses of PTP and thermoplastic layers are, respectively, 1.8 and $0.5 \mu \mathrm{m}$. (a) 3D surface plot, (b) relief profile.

the best values of spatial frequency of the two-layer carrier $\left(1250 \mathrm{~mm}^{-1}\right.$ for the analyzed sample, corresponding to a fringe spacing of $\approx 0.8 \mu \mathrm{m}$ ). The relief height is about $80 \mathrm{~nm}$. The surface structuration observed is similar to the one previously evidenced in the case of the one-layer PTP carrier. The possibility of multiple recording has been investigated. The maximum diffraction efficiency $(10 \%)$ is maintained after ten consecutive record-erase cycles with a diffraction grating having a spatial frequency equal to $1000 \mathrm{~mm}^{-1}$.

\section{Conclusion}

This paper presents a complete study, from the material synthesis to its use as holographic recording media. Firstly, syntheses and characterizations of carbazolic monomers and copolymers were carried out. Their chemical structure was examined implementing various analytical techniques. The syntheses yielding carbazole containing copolymers are rather complex from the first step, the monomer synthesis, to the final copolymer elaboration. By combining NMR and IR techniques, it was possible to firmly assess the structure and the composition of the various participants in the chain of reactions. The implemented approach gives access to a precise characterization of copolymers; this is a crucial point when considering their wide use as photosensitive or electrophotosensitive materials to record holograms. The procedure may be applied to any carbazole containing polymer or copolymer. Secondly, reversible holographic recordings were carried out with the synthesized materials. Hologram recording via a photothermoplastic (PTP) process provides the possibility of hologram recording and reading practically in real time $(\sim 3 \mathrm{~s})$. The comparison between one- and two-layer PTP carriers showed that the use of two independent layers of thermoplastic and photopolymer is advantageous, giving rise to better results in terms of resolution and sensitivity. Experimentally, it is possible to record and read holograms with a diffraction efficiency of $10 \%$. The obtained holographic characteristics of CEM1OMA photopolymer are very close to those of well-known poly(vinylcarbazole) [9]. Its advantage consists however, in a better compatibility with the thermoplastic layer of styrene and butylmethacrylate. As a result, the carrier is more stable during the PTP recording process allowing more than ten write/read cycles. Even if the values obtained can be further improved, those results have permitted to characterize the holographic properties of the system and to give evidence of the formation of a relief by AFM analysis. After holographic exposure and in the dark areas, the attraction of the positive charges located at the surface by the negative charges underneath leads, during thermal treatment, to a deformation of the thermoplastic material. The examination of the surface showed that the charges imprint could be observed. Channels made of aligned and closely juxtaposed holes are observed in the lower parts of the pattern. The depth of the relief is increasing with charges concentration. The difference between the one-layer and the two-layer PTP carriers resides in the fact that in the latter case, diameter and height of these holes are much smaller.

These analyses represent the first indispensable steps of a larger study devoted to the comprehension of charge transport and photoinduced transformation mechanisms occurring during optical information recording and processing, especially with hologram formation.

\section{Acknowledgments}

The authors gratefully acknowledge the Alliance Française of Moldova for partial financial support of this work. They thank Dr. Ion Andries, Moldova State University, for his valuable assistance in holographic experiments and their interpretation.

\section{References}

[1] H. Hoegl, "On photoelectric effects in polymers and their sensitization by dopants," Journal of Physical Chemistry, vol. 69, no. 3, pp. 755-766, 1965.

[2] J. V. Grazulevicius, P. Strohriegl, J. Pielichowski, and K. Pielichowski, "Carbazole-containing polymers: synthesis, properties and applications," Progress in Polymer Science, vol. 28, no. 9, pp. 1297-1353, 2003.

[3] K. Meerholz, B. L. Volodin, Sandalphon, B. Kippelen, and N. Peyghambarlan, "A photorefractive polymer with high optical 
gain and diffraction efficiency near 100\%," Nature, vol. 371, no. 6497, pp. 497-500, 1994.

[4] Y. Zhang, C. A. Spencer, S. Ghosal, M. K. Casstevens, and R. Burzynski, "Thiapyrylium dye sensitization of photorefractivity in a polymer composite," Applied Physics Letters, vol. 64, no. 15, pp. 1908-1910, 1994.

[5] D. P. West, M. D. Rahn, C. Im, and H. Bässler, "Hole transport through chromophores in a photorefractive polymer composite based on poly(N-vinylcarbazole)," Chemical Physics Letters, vol. 326, no. 5-6, pp. 407-412, 2000.

[6] I. K. Moon, C.-S. Choi, and N. Kim, "Synthesis and characterization of novel photoconducting carbazole derivatives in main-chain polymers for photorefractive applications," Polymer, vol. 48, no. 12, pp. 3461-3467, 2007.

[7] V. Bivol, S. Robu, G. Dragalina, L. Bostan, A. Prisacari, and A. Coban, "New photoresists from carbazol-containing photopolymers," in Applications of Photonic Technology, vol. 4087 of Proceedings of SPIE, pp. 754-759, 2000.

[8] E. Chilat, Synthèse, caractérisation et durabilité de systèmes photostructurables destinés à l'holographie, Ph.D. thesis, Université Blaise Pascal, Clermont-Ferrand, France, 2008.

[9] I. Banyasz, "The effects of the finite spatial resolution of thermoplastic recording materials on the holographic image," Journal de Physique, vol. 3, no. 7, pp. 1435-1444, 1993.

[10] R. Collier, C. Burckhardt, and L. Lin, Optical Holography, Academic Press, NJ, USA, 1971.

[11] N. Barba, G. Dragalina, O. Russu, and L. Vlad, USSR Patent cl C07d 209/82, no. 1634007, 1990.

[12] Aldrich FT-IR Digital Spectral Library, Condensed Phase, Thermo-Scientific.

[13] D. Lin-Vien, N. B. Colthup, W. G. Fateley, and J. G. Grasselli, The Handbook of IR and Raman Characteristic Frequencies of Organic Molecules, Academic Press, San Diego, Calif, USA, 1991.

[14] L. I. Berger, Semiconductor Materials, CRC Press, Boca Raton, Fla, USA, 1996.

[15] V. Bivol and S. Robu, in Proceedings of the 5th International Conference on Frontiers of Polymers and Advanced Materials, pp. 373-385, Poznan, Poland, 1999. 


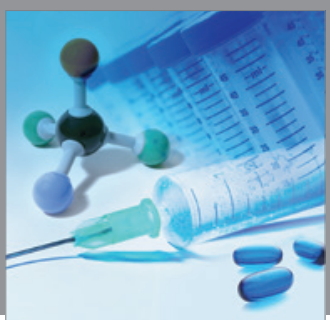

International Journal of

Medicinal Chemistry

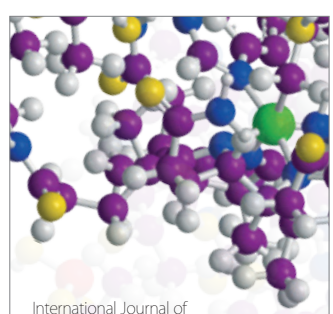

Carbohydrate Chemistry

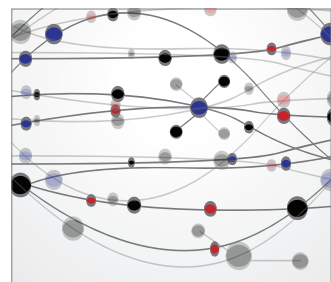

The Scientific World Journal
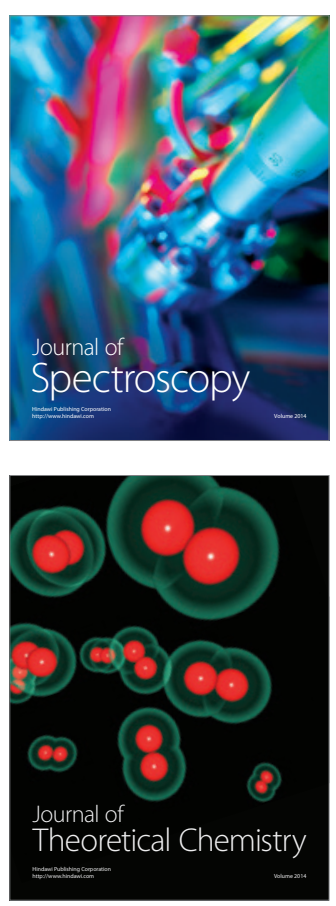
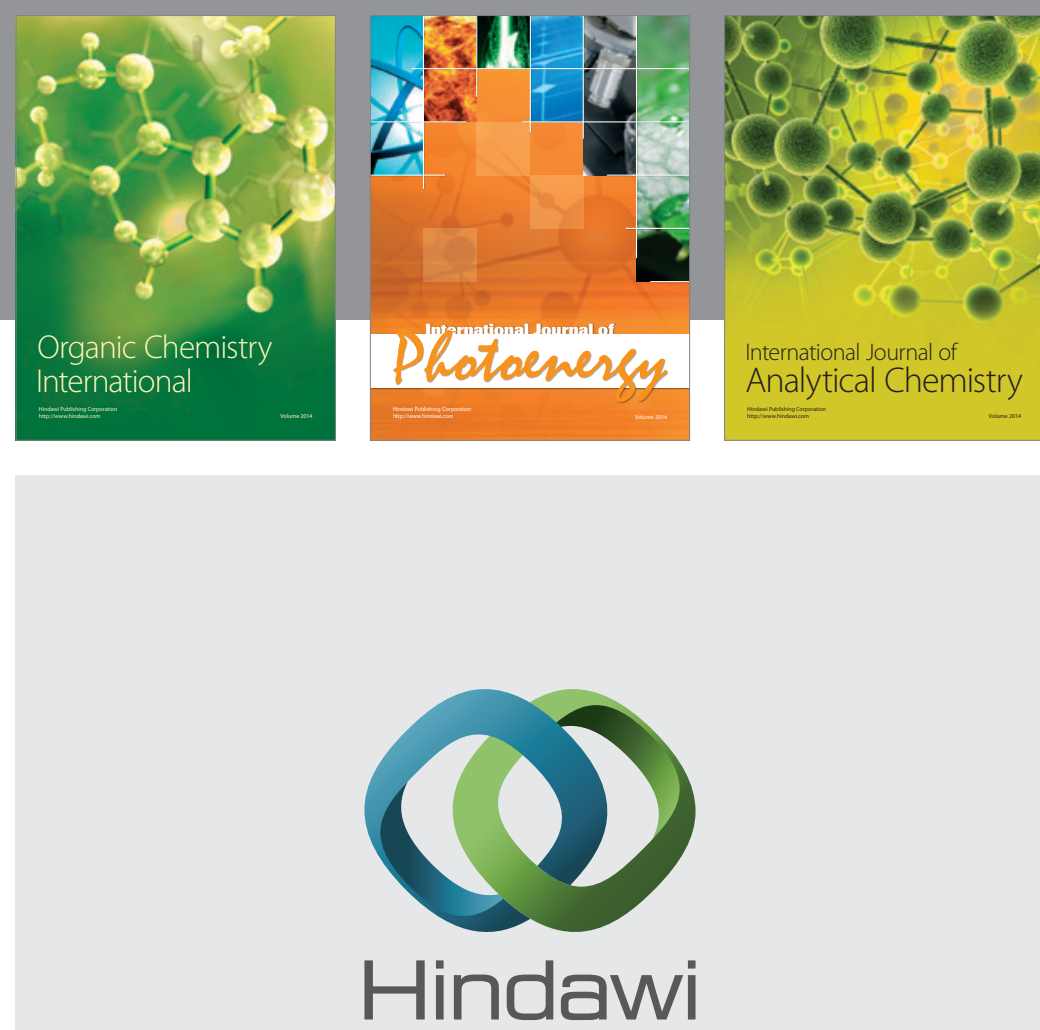

Submit your manuscripts at

http://www.hindawi.com
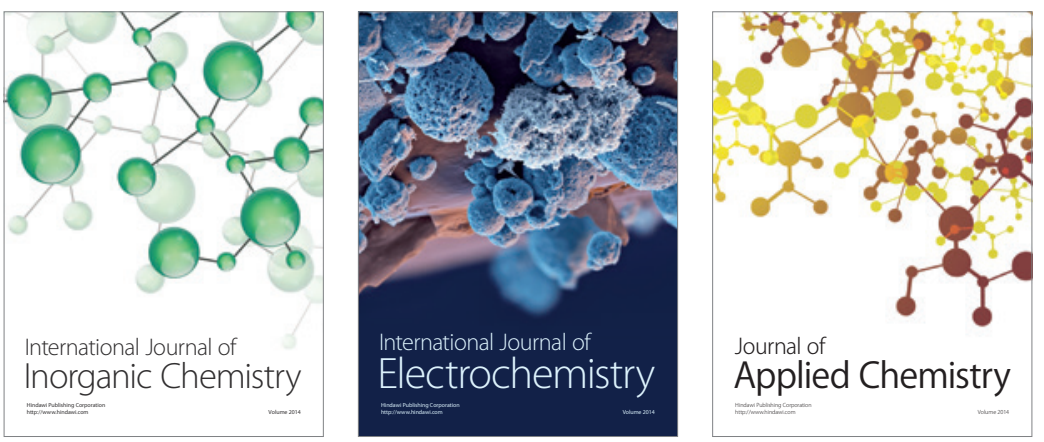

Journal of

Applied Chemistry
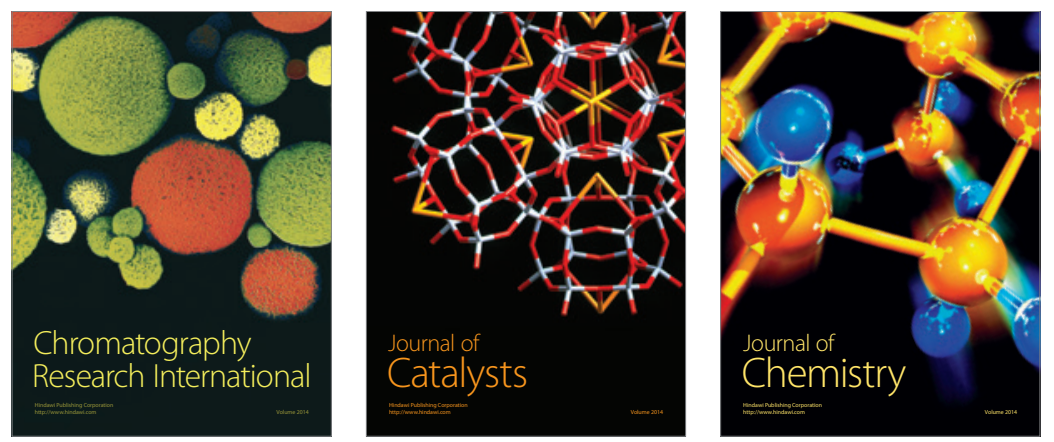
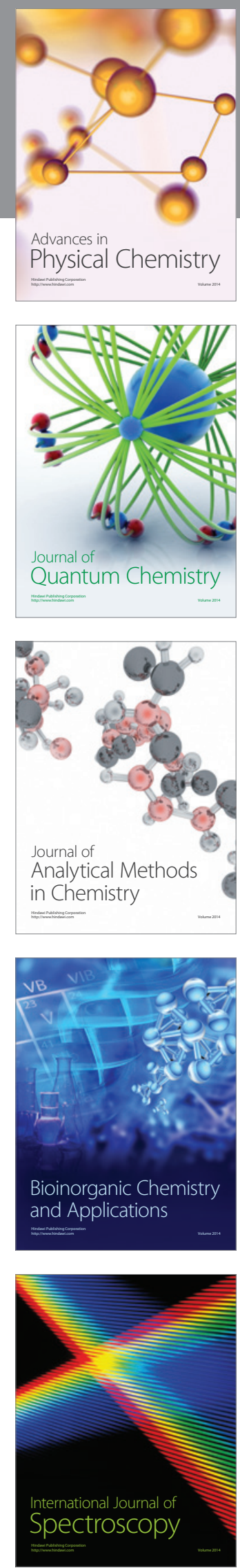